\title{
The Distribution of Nicotinic Acid in the Rice Grain
}

\author{
By J. J. C. HINTON AND B. SHAW \\ Research Association of British Flour Millers, Cereals Research Station, St Albans
}

(Received 2 I $\mathscr{f} u l y$ 1953)

For the more important members of the B group of vitamins most of the world's human population is mainly dependent upon one or other of the cereal grains. The cereal grain is a complex structure, and it was already implied that the vitamins are not uniformly distributed within the grain when it was first suggested that beriberi was a nutritional disease (Grijns, I900-I I) produced by the consumption of rice from which the outer layers had been removed by milling (Braddon, 1907; Fraser \& Stanton, I909). This was before Funk had separated from these outer layers, the rice polishings, an active principle effective in curing polyneuritis in birds, and had first suggested the existence of a group of such active substances to be called 'vitamines'. The unequal distribution was fully recognized by Bréaudat $\&$ Denier (I9I I), who found that rice polishings were more effective than unmilled grain in curing beriberi. Chick \& Hume (I917) later showed that the germ both of rice and wheat was more effective than the branny layers. More recently, Altson and Simpson observed that embryos dissected from rice contained approximately half of the total vitamin $B_{1}$ of the grain (Altson, I94I). By detailed dissection, first of wheat and later of rice, one of us has found that the greatest concentration of vitamin $B_{1}$ occurred in the scutellum. In rice the latter contained $44-50 \%$ of the total vitamin $B_{1}$ of the grain (Hinton, 1948-9).

Aykroyd \& Swaminathan (1940) demonstrated that during the milling of rice both nicotinic acid and vitamin $B_{1}$ were removed in the polishings. Wang, Yang \& Ch'eng (1950), employing dissection methods, found that the greater part of the nicotinic acid in the rice grain was present not in the scutellum but in the outer layers, $73 \%$ being in the pericarp and aleurone layer. In wheat $82 \%$ and in maize $60 \%$ of the nicotinic acid of the grain has been found in the aleurone layer (Heathcote, Hinton $\&$ Shaw, I952).

This paper describes a detailed study of the distribution of nicotinic acid in two types of rice.

\section{EXPERIMENTAL}

Material. Two samples of rice were examined. One, drawn from commerce, was of Egyptian origin but of unknown variety. It was very uniform, a short, broad, white grain, well developed and in excellent condition.

The second was an Indian variety A.S.D.3, a longer, narrower, red-skinned grain. We understand that this is not at present much used in agriculture; it was chosen because it was a well-developed grain very suitable for dissection. 
Dissection of the grain. The structure of the rice grain is similar to that of the other cereals and has been described by several workers (e.g. Winton \& Winton, 1932). In brief it consists of the starch-bearing endosperm containing mainly starch and protein; the aleurone layer, the outermost layer of the endosperm, containing no starch but relatively rich in oil and protein; the embryo or germ, comprising scutellum and embryo proper; and the skins, which cover the whole, the seed coat and the various layers of the pericarp.

In the embryo the coleorhiza and epiblast are well developed and can readily be separated from the plumule and radicle. The embryo was not so subdivided for our work, but was removed as a whole. By placing the grain in a moist atmosphere for 2 or 3 days the embryo was allowed to swell, so that after removal of the covering layers it was in a suitable condition for dissection and removal. The scutellum was then separated in its moist condition from the layer of crushed empty endosperm cells on which it rests, and the grain was thereafter allowed to return to an air-dry condition.

The layers of the pericarp are fragile and not well defined, and attempts to separate them quantitatively from one another and from the aleurone layer were unsuccessful. With some difficulty small amounts of aleurone layer and of part of the pericarp were obtained and assayed separately. For the quantitative dissection, however, pericarp, testa and aleurone layer were removed together by the gentle action of a small scraper having a rounded smooth edge. The starchy endosperm was subdivided by scraping with a small knife, so that a thin outer layer was removed, corresponding to about $6 \%$ of the grain. This is an arbitrary subdivision having no anatomical basis.

The dissection was carried out under a binocular microscope, the grain being held in a small pin vice. As dissecting instruments, surgical needles of various sizes were used, together with tools shaped from steel or nickel wire.

The parts into which the grains were dissected, therefore, were: pericarp, testa and aleurone layer, outer endosperm, inner endosperm, embryo, and scutellum.

Assay of nicotinic acid. Nicotinic acid in the material was assayed microbiologically. The procedure, the same as that previously described (Heathcote et al. 1952), was briefly as follows:

(a) Preparation of the hydrolysates. Typical grains $(5 \mathrm{~g})$ were coarsely ground in a coffee mill and suspended in $5 \circ \mathrm{ml}$. $\mathrm{N}-\mathrm{HCl}$.

Dissected fractions, 2-200 $\mathrm{mg}$, were suspended in $\mathrm{I} \mathrm{ml} . \mathrm{N}-\mathrm{HCl}$.

All samples were hydrolysed by heating to $120^{\circ}$ for $20 \mathrm{~min}$. The $50 \mathrm{ml}$. extracts were then prepared for assay as described by Barton-Wright (1945). The I ml. extracts were buffered by the addition of $0.04 \mathrm{ml}$. of $2.5 \mathrm{M}$-sodium acetate and partially neutralized with $\mathrm{I} \mathrm{ml} . \mathrm{N}-\mathrm{NaOH}$. The $\mathrm{pH}$ was adjusted to 4.5 when any solid matter was to be removed and thereafter to 6.8 before making to a volume chosen to give approx. $0.05 \mu \mathrm{g}$ nicotinic acid $/ \mathrm{ml}$.

(b) Effect of lipid. Under some conditions the presence of lipid material in the assay solutions has been found to influence the growth of bacteria, and some analysts have removed lipid material as a standard procedure before assay. We have found removal 
of lipids to have no appreciable effect with rice, as the following relative figures illustrate:

Light petroleum extraction of material from A.S.D. 3 before hydrolysis:

(1) Pericarp and aleurone fraction unextracted

(2) Pericarp and aleurone fraction extracted

(3) Hydrolysate from (z) extracted with ethyl ether

roo

(4) Whole grain unextracted

(5) Whole grain extracted

I00 (I) taken as I00

$100)$

I00 $\left.9 \mathrm{I}_{5}\right\}(4)$ taken as 100

Effect of solvent extraction of hydrolysate of whole rice A.S.D.3:

(I) Unextracted hydrolysate

(2) Chloroform-extracted hydrolysate

(3) Ethyl ether-extracted hydrolysate

100)

100 (1) taken as 100

96)

Extraction of lipid was therefore omitted from the procedure.

(c) Nature of the nicotinic acid estimated. A large proportion of the nicotinic acid present in cereal grains exists in a bound form that is not available to the bacteria used in the assay, but is to higher animals, probably including man. Hydrolysis with $\mathrm{N}-\mathrm{NaOH}$ is accepted as the means of liberating fully this bound nicotinic acid, but it is an inconvenient procedure when much starch is present. A comparison of $\mathrm{N}-\mathrm{HCl}$, a convenient reagent, and $\mathrm{N}-\mathrm{NaOH}$ for hydrolysis of whole rice, endosperm, and pericarp with aleurone fractions gave results differing by not more than $7 \%$. It is concluded that the figures quoted as results express the total nicotinic acid.

(d) Assay procedure. The culture medium described by Barton-Wright (I945) was used; each tube was inoculated with one drop of a standard-sized inoculum of Lactobacillus arabinosus. After $72 \mathrm{~h}$ incubation at $37^{\circ}$ the acid formed was titrated with O. I N-NaOH.

A small series of comparative assays with $L b$. mesenteroides was run on representative material, to serve as a check on the suitability of the culture being used: the figures are expressed in terms of values for the $L b$. arabinosus taken as 100 .

$\begin{array}{lcc} & L b . \text { arabinosus } & L b . \text { mesenteroides } \\ \text { Whole rice A.S.D.3 } & 100 & 93 \cdot 8 \\ \text { Endosperm fraction } & 100 & 1 \mathrm{II} \\ \text { Pericarp and aleurone fraction } & 100 & 103\end{array}$

The results from the two organisms are in reasonable agreement.

The criteria suggested by Wood were used to judge the validity of each assay, and the results were calculated by the slope-ratio method (Wood, 1946).

Though the assays were completed as soon as the material was available it sometimes required 2 weeks to collect sufficient of the dissected fractions. This delay would have no appreciable effect on the results, however, since it was observed during the preliminary experimental work that the value found both for dissected fractions and for hydrolysed extracts did not change appreciably during periods of up to 6 weeks under the storage conditions at $4^{\circ}$.

\section{RESULTS}

The results are presented in Tables $\mathrm{r}$ and 2. They are based on air-dry weight which under our laboratory conditions is equivalent to $89 \pm \mathrm{I} \%$ of dry matter.

The pericarp and aleurone fraction was further subdivided on a very small scale, the figures obtained being given in Table 2 . 
Table $\mathrm{I}$. Distribution of nicotinic acid in dissected fractions of the rice grain

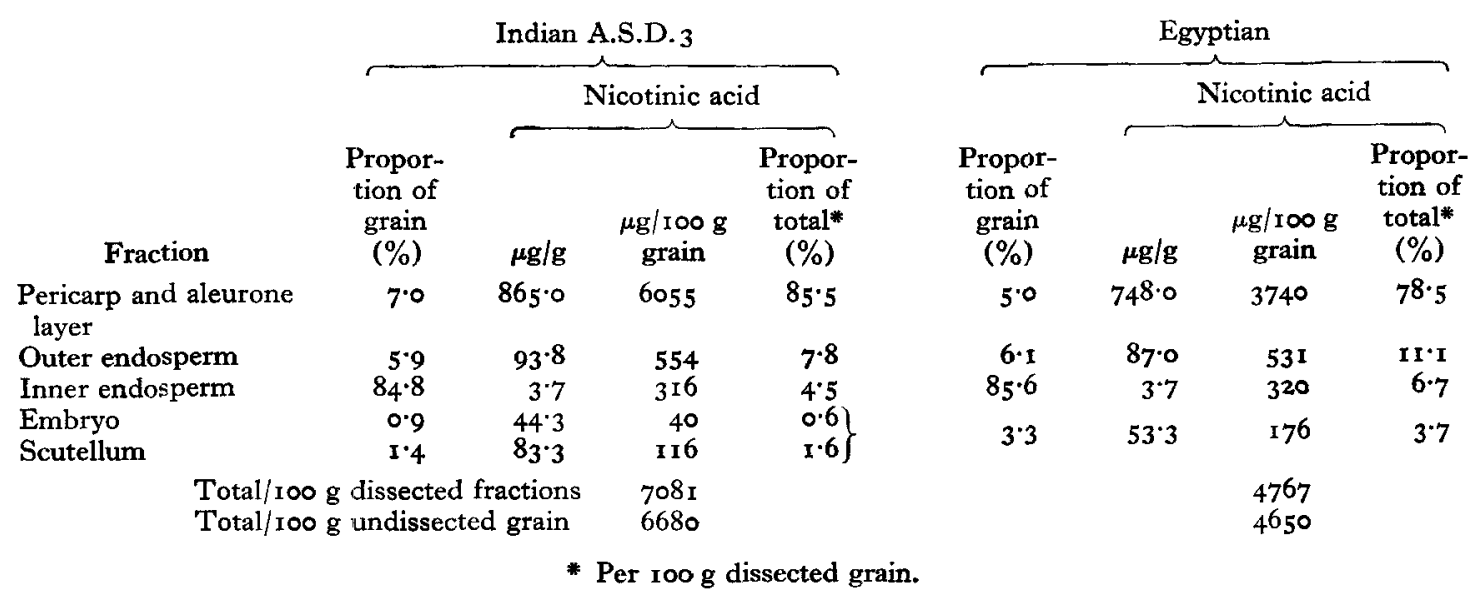

Table 2. Distribution of nicotinic acid in subdivisions of the pericarp and aleurone fraction of Indian rice A.S.D.3

$\begin{array}{cccc}\text { Fraction } & \begin{array}{c}\text { Proportion of } \\ \text { grain }(\%)\end{array} & \begin{array}{c}\text { Picotinic acid } \\ (\mu \mathrm{g} / \mathrm{g})\end{array} & \begin{array}{c}\text { Proportion of } \\ \text { total nicotinic } \\ \text { acid } \\ (\%)\end{array} \\ \begin{array}{c}\text { Outermost part } \\ \text { of pericarp }\end{array} & 1.4^{*} & 156 & 3.1^{*} \\ \begin{array}{c}\text { Remainder of } \\ \text { pericarp }\end{array} & 0.9 \dagger & 156 \dagger & 2.0 \dagger \\ \text { Aleurone layer } & 4.7^{*} & 1200 & 80.5^{*}\end{array}$

* Approximate figures.

+ Figures based on assumption and calculation, and not the results of direct analysis.

\section{DISCUSSION}

The sum of the amounts of nicotinic acid found in the dissected fractions agrees well with the total found in the undissected grain.

In the two samples examined the general pattern of distribution of the nicotinic acid is similar to that already reported for wheat, and it may be of interest to compare the more important details. Thus 78.5 and $85.5 \%$ of the total nicotinic acid of the grain is found in the pericarp and aleurone fraction, compared with $82 \%$ in the aleurone layer of wheat. The content of nicotinic acid in the pericarp and aleurone fractions, $74^{8}$ and $865 \mu \mathrm{g} / \mathrm{g}$, is also similar to that of the aleurone layer of wheat, 613 and $74 \mathrm{I} \mu \mathrm{g} / \mathrm{g}$ for two varieties; that in the aleurone layer of A.S.D. $3,1200 \mu \mathrm{g} / \mathrm{g}$, is appreciably higher than the nicotinic acid in dried brewer's yeast, previously recorded as the richest natural source, and approaches in value the concentrates prepared from rice polishings (Bacharach, I940-r).

Similarly, there is a marked gradient in the starch-bearing endosperm, which is probably somewhat steeper than in wheat. The outer $6.5 \%$ in rice is nearly thirty times as rich as the central part, in which the concentration is approximately twothirds that of the same region in wheat. The pericarp of rice has not been assayed as 
a whole, but it appears that its concentration is about six times that in the pericarp of wheat. These comparisons are made between grain samples that have nearly the same total concentration of nicotinic acid.

There are slight differences between the two samples of rice. In the Egyptian rice the pericarp and aleurone fraction amounts to $5 \%$ of the grain, compared with $7 \%$ in A.S.D. 3. This is due in part to the different grain shape, which results in a different ratio of surface area to volume. When the difference in total nicotinic acid of the grain is taken into account, the pericarp and aleurone fraction of the Egyptian rice is seen to be relatively richer in nicotinic acid than is A.S.D.3. This, however, is more than counterbalanced by the smaller proportion of this fraction in the Egyptian rice, with the result that it accounts for $78.5 \%$ of the total, compared with $85.5 \%$ in A.S.D.3.

The concentration in the endosperm is almost the same in each of the samples, so that in the Egyptian it is proportionately higher; in it the endosperm contains $17.8 \%$ of the total, compared with $12.3 \%$ in the Indian.

Embryo and scutellum were not separated in the Egyptian, as they were in A.S.D.3, but the germ as a whole is proportionately richer in nicotinic acid in the former and also comprises a slightly greater proportion of the grain. In both it is an insignificant source of nicotinic acid.

There is no suggestion in these figures that the distribution is significantly different in red-skinned and white-skinned varieties.

Our results are in good agreement with those of Wang et al. (1950), which only became available to us after the preparation of this paper. With the exception of the inner endosperm the nicotinic-acid content of their fractions, which were defined similarly to ours, was $20-25 \%$ lower than those reported here. The inner endosperm contained $8 \mu \mathrm{g} / \mathrm{g}$ compared with our figure of $3.7 \mu \mathrm{g} / \mathrm{g}$ and the endosperm as a whole accounted for $22 \%$ of the total compared with our average figure of $13 \%$. The pericarp and aleurone layer contained correspondingly less of the total, $73 \%$ compared with the average of $82 \%$.

\section{Comparison of the distribution of nicotinic acid and thiamine}

The distribution of nicotinic acid in the rice grain, as in the other cereals so far examined, is different from that of thiamine. The diagrams of Fig. I compare the distribution of these two vitamins based on the average figures from two samples of rice. Nearly half of the thiamine and only $2 \%$ of the nicotinic acid was found in the scutellum; in the germ as a whole there were $58 \%$ of the thiamine and $3 \%$ of the nicotinic acid.

On the other hand, $81 \%$ of the nicotinic acid and only $36 \%$ of the thiamine were in the pericarp and aleurone fraction. The endosperm contained 7.8 and $15 \%$ of the thiamine and nicotinic acid, respectively.

In principle, therefore, it is possible to separate the major part of these two vitamins of the grain mechanically by simply separating the anatomical structures concerned. In the present-day practice of milling rice this separation does not take place because the germ is readily dislodged from the grain once the pericarp and aleurone layers are removed. Processes with a gentler action, under which the germ tends to be 
retained in the grain, have been proposed as a means of improving the nutritional value of the milled rice and would certainly have the effect of producing an increased thiamine content. The achievement of a satisfactory nicotinic-acid content, however, is an entirely separate problem, depending upon the retention of the aleurone layer, and as such would require its own solution.

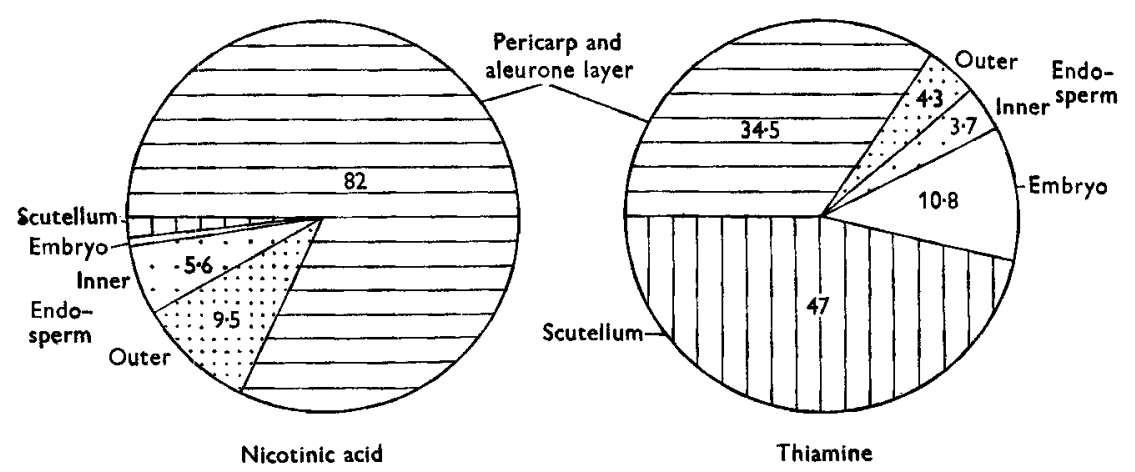

Fig. I. The distribution of nicotinic acid and thiamine in the rice grain.

\section{SUMMARY}

I. The grain of two varieties of rice has been dissected by hand into its structural parts, the endosperm being arbitrarily subdivided, and the nicotinic acid therein determined by microbiological assay.

2. Approximately $8 \mathrm{r} \%$ of the nicotinic acid of the grain was found in the pericarp and aleurone-layer fraction, the aleurone layer itself containing $1200 \mu \mathrm{g} / \mathrm{g}$.

3. The endosperm contained $15 \%$ of the nicotinic acid of the grain, with a notable decrease in gradient from the outer to the inner portions.

4. The pattern of distribution of nicotinic acid is compared with that of thiamine, of which $50 \%$ is found in the scutellum. The distribution of nicotinic acid is similar in red- and white-skinned varieties and resembles that found in wheat.

The Indian rice A.S.D.3 was obtained through the courtesy of Dr K. Ramiah, Director, Central Rice Research Institute, Cuttack.

\section{REFERENCES}

Altson, R. A. (194I). Malay. agric. F. 29, 127.

Aykroyd, W. R. \& Swaminathan, M. (1940). Indian F. med. Res. 27, 667.

Bacharach, A. L. (r940-r). Nutr. Abstr. Rev. Io, 459.

Barton-Wright, E. C. (1945). Analyst, 70, 283.

Braddon, W. L. (1907). Causes and Prevention of Beri-Beri. London. Quoted by Medical Research Council: Committee Appointed jointly by the Lister Institute and the Medical Research Council (1924). Spec. Rep. Ser. med. Res. Coun., Lond., no. 38 (revised).

Bréaudat, L. \& Denier (r9r I). Ann. Inst. Pasteur, 25, I67.

Chick, H. \& Hume, E. M. (1917). Proc. roy. Soc. B, 90, 44.

Fraser, H. \& Stanton, A. T. (r909). Quoted by Medical Research Council: Committee Appointed jointly by the Lister Institute and the Medical Research Council (1924). Spec. Rep. Ser. med. Res. Coun., Lond., no. $3^{8}$ (revised). 
Grijns, G. (1900-I I). Quoted by Nelson, E. M. (1939). In The Vitamins, p. 127. [Council on Pharmacy and Chemistry and Council on Foods of the American Medical Association, editors.] Chicago: American Medical Association.

Heathcote, J. G., Hinton, J. J. C. \& Shaw, B. (1952). Proc. roy. Soc. B, 139, 276.

Hinton, J. J. C. (1948-9). Brit. F. Nutr. 2, 237.

Wang, Y. L., Yang, K. C. \& Ch'eng, S. M. (1950). Chung Kuo Ko Hsueh (Peking), 1, 1, 99.

Winton, A. L. \& Winton, K. B. (1932). The Structure and Composition of Foods. New York: John Wiley and Son.

Wood, E. C. (1946). Analyst, 7I, I.

\title{
Some Water-soluble Vitamins in the Sweat of Tropically Acclimatized European Men
}

\author{
By J. W. H. LUGG \\ Department of Biochemistry, University of Malaya, Singapore \\ AND F. P. ELLIS \\ Medical Research Council's Royal Naval Tropical Research Unit, Singapore
}

(Received 18 August 1953)

The earlier literature on the concentrations of ascorbic and dehydroascorbic acids in human sweat and on the dermal losses has been reviewed by Kirch, Cornbleet \& Bergeim (1943), Mickelsen \& Keys (I943), Sargent, Robinson \& Johnson (1944), Shields, Johnson, Hamilton \& Mitchell (1945) and Tennent \& Silber (1943), who all, in turn, contributed their own findings. General reviews of dermal losses of ascorbic and dehydroascorbic acids and other water-soluble vitamins are given in Nutrition Reviews (Anonymous, 1946) and by List (1948).

The more recent work has not confirmed the earlier view that the loss of ascorbic acid in profuse sweating may be considerable. There is, however, appreciable conflict between some of the conclusions drawn from two of the more recent studies by Kirch et al. (1943) and by Shields et al. (1945). The latter encountered a decrease in the ascorbic-acid and an increase in the dehydroascorbic-acid content of sweat after an increase in the ascorbic-acid intake, instead of an increase in both, as encountered by the former workers: further, for subjects receiving large dietary supplements of L-ascorbic acid and sweating under rather similar conditions, they reported a dehydroascorbic-acid level in the sweat only about one-eighteenth as high as that encountered by the former workers. In these studies the subjects were exposed periodically to artificial climatic conditions, which usually simulated hot dry or hot humid climates, but they lived in much more temperate, natural climates when not taking part in the experiments. The subjects studied by Shields et al. (1945), however, spent a considerable part of their daily lives in the artificial climates.

An opportunity arose between programmes of work in progress at the Royal Naval 